\title{
LA GUERRE CIVILE ESPAGNOLE, ENJEUX HISTORIOGRAPHIQUES ET PATRIMOINE POLITIQUE
}

François Godicheau

\section{Presses de Sciences Po (P.F.N.S.P.) | «Vingtième Siècle. Revue d'histoire »}

2015/3 $\mathrm{N}^{\circ} 127$ | pages 59 à 75

ISSN 0294-1759

ISBN 9782724634341

Article disponible en ligne à l'adresse :

http://www.cairn.info/revue-vingtieme-siecle-revue-d-histoire-2015-3-page-59.htm

\section{!Pour citer cet article :}

François Godicheau, « La guerre civile espagnole, enjeux historiographiques et patrimoine politique », Vingtième Siècle. Revue d'histoire 2015/3 ( $\left.\mathrm{N}^{\circ} 127\right)$, p. 59-75.

DOI 10.3917/ving.127.0059

Distribution électronique Cairn.info pour Presses de Sciences Po (P.F.N.S.P.).

(C) Presses de Sciences Po (P.F.N.S.P.). Tous droits réservés pour tous pays.

La reproduction ou représentation de cet article, notamment par photocopie, n'est autorisée que dans les limites des conditions générales d'utilisation du site ou, le cas échéant, des conditions générales de la licence souscrite par votre établissement. Toute autre reproduction ou représentation, en tout ou partie, sous quelque forme et de quelque manière que ce soit, est interdite sauf accord préalable et écrit de l'éditeur, en dehors des cas prévus par la législation en vigueur en France. Il est précisé que son stockage dans une base de données est également interdit. 


\section{La guerre civile espagnole, enjeux historiographiques et patrimoine politique}

François Godicheau

En proposant une analyse pugnace de la guerre civile, François Godicheau revisite en même temps cinquante ans d'histoire de l'Espagne. En effet, le récit canonique forgé lors de la transition démocratique, qui vient alors contrer la propagande franquiste, fonctionne comme un mythe, au sens barthien du terme, fondant et légitimant un régime. Cependant, l'offensive idéologique des néofranquistes, les mouvements mémoriels et la crise économique et politique ont déstabilisé ce métarécit et en ont montré les limites. L'auteur nous livre un riche exemple des usages politiques du passé et de ses mises en récit.

Au milieu des années 1990, il semblait que l'histoire de la guerre civile espagnole était à la fois relativement pacifiée et de moins en moins pratiquée par les historiens ${ }^{1}$. Aujourd'hui, on ne peut que constater la présence très importante de ce passé dans le présent, même si les problèmes de l'Espagne sont bien des problèmes du présent. Pendant ce temps, et depuis le soixante-dizième anniversaire du début de la guerre en 2006, les livres sur celle-ci continuent à se publier, et pas seulement les essais

(I) Les historiens, qui avaient écrit des livres marquants autour du cinquantenaire de 1986, étaient peu ou prou « passés à autre chose ». C'était le cas d'historiens importants pour ce champ, comme Julio Aróstegui Sánchez et Julián Casanova qui, dix ans plus tard, sont revenus sur la question. historiques : romans, mémoires, livres hybrides mêlant fiction et bouts d'archives, films, feuilletons, expositions. Il y a là une véritable industrie qui n'a plus besoin des éphémérides (1986, 1996 et 2006) pour multiplier productions et ventes. Cette profusion de narrations à propos de laquelle on a prétendu que l'événement avait donné lieu à plus de livres que la Seconde Guerre mondiale serait plutôt dissuasive pour celui qui aborde ce champ ${ }^{2}$. Les publications se multiplient au point de rendre la veille bibliographique très difficile, mais en dépit d'un large consensus historiographique au sein de l'Université, on ressent un décalage entre la très grande tranquillité des débats entre historiens professionnels et la passion avec laquelle ce passé est convoqué dans la société actuelle. Polémiques, scandales, accusations croisées rythment une chronique dans laquelle on voit intervenir professionnels de

(2) D'après Enrique Moradiellos, il s'agit d'une idée de l'historien anglais Paul Preston qui, en 1986, dans son livre sur la guerre d'Espagne intitulé The Spanish Civil War 1936-1939 (Londres, Weidenfeld \& Nicoloson), situait le chiffre à 15000 livres. Le travail de bibliographie du Conseil supérieur de la recherche scientifique (Consejo superior de investigaciones científicas, CSIC, équivalent du Centre national de la recherche scientifique, CNRS, en France) en 1996 comptait 1848 livres et 1749 articles entre 1975 et 1996 : Julio Aróstegui Sánchez (dir.), BIHES : bibliografías de bistoria de España, Madrid, Editorial Consejo superior de investigaciones científicas (CSIC)/ Centro de información y documentación científica (CINDOC), Departamento de ciencias humanas, 1996, vol. 6 et 7 . 
l'histoire, associations, politiques, journalistes, écrivains et essayistes divers.

Cette situation de cohabitation de la bonace historiographique avec la tempête mémorielle oblige à examiner l'historiographie de la guerre dans ses rapports avec le débat public. L'écriture de l'histoire de la guerre d'Espagne est profondément conditionnée par des politiques de mémoire vieilles de plusieurs décennies, qui ont été décisives au moment de la fondation des institutions actuelles de la monarchie constitutionnelle ${ }^{1}$. La légitimité de ces institutions repose en grande partie sur un récit historique hégémonique de la période des années 1930, forgé pendant la «transition démocratique $\gg$. Celle-ci constitue le pendant, l'inverse de la guerre dans une échelle politique du bien et du mal qui fait de la « guerre civile» la catastrophe absolue, et de la «transition» un modèle de réussite, le tout dessinant l'histoire d'une rédemption de l'Espagne, d'un cheminement difficile et obscur jusqu'à la lumière, et une réussite qui rachèterait les souffrances passées.

Il existe ainsi un métarécit de la guerre qui s'articule autour de la caractérisation du conflit comme une « folie collective », dont le récit est censé conjurer la répétition, suivant la logique traditionnelle de l'histoire magistra vite ${ }^{2}$. Le récit historique devient un cri de «plus jamais ça ! » et c'est presque ce qui fonde sa légitimité sociale. Cette conception se conjugue avec l'idée du progrès de l'Espagne sur le chemin de la modernité et l'affirmation d'une nation enfin prospère, démocratique et « européenne ». Les

(I) Paloma Aguilar Fernández, Políticas de la memoria y memorias de la política : el caso español en perspectiva comparada, Madrid, Alianza, 2008.

(2) Leopoldo Moscoso, « En qué consiste pensar históricamente ? », et Elías Palti, «Pensar históricamente en una era postsecular : o el fin de los historiadores después del fin de la historia », dans Pablo Sánchez León et Jesús Izquierdo Martín (coord.), El fin de los historiadores : pensar históricamente en el siglo XXI, Madrid, Siglo XXI, 2008, p. 3-26 et p. 27-40. valeurs politiques qui président à la narration sont alors celles d'une démocratie identifiée, jusqu'à ce que les «indignés » s'exclament, le 15 mai 2011, «ils appellent ça démocratie, mais ça n'en est pas une », au régime né avec la Constitution de 1978.

\section{Les conditions de production d'un récit hégémonique}

La principale justification morale des modalités de la transition vers la démocratie était l'idée de la réconciliation entre deux Espagne naguère déchirées par un combat sans merci. La mobilisation du passé était en effet nécessaire, car la succession des événements depuis 1975 pouvait être susceptible d'une lecture moins complaisante que l'on peut résumer ainsi : avec la mort du général Franco en novembre 1975, le pouvoir, très inquiet de l'exemple portugais où la chute de la dictature sœur semblait vouloir se prolonger en révolution sociale, tenta quelques gestes d'ouverture timides, mais sa réaction extrêmement violente face à des mobilisations collectives massives, radicales et menaçant de déborder le Parti communiste (Partido comunista de España, PCE) lui-même, ruina cette première stratégie. Face aux mobilisations populaires de plus en plus nombreuses, le dirigeant le plus dynamique de la Phalange ${ }^{3}$, parti unique, chef du gouvernement à la fin du printemps 1976, Adolfo Suárez, proposa un plan de réforme du régime. Il parvint à un accord pendant l'été avec le secrétaire général du Parti communiste, Santiago Carrillo, qui promit en outre de consolider le processus par un pacte social. Suárez trouva un autre partenaire important en la personne de Felipe González, dirigeant d'un Parti socialiste ouvrier espagnol (Partido socialista obrero español, PSOE) pratiquement refondé avec

(3) Une note sur la Phalange figure en page 10 de ce numéro. 
l'argent et les conseils du Parti social-démocrate d'Allemagne (Sozialdemokratische Partei Deutschlands, SPD) de Willy Brandt ${ }^{1}$ (via la Fondation Friedrich-Ebert) sur une ligne d'extrême modération, dans le cadre de la stratégie de contention de l'OTAN. Ce consensus en faveur d'une transition en douceur fut accepté par les procurateurs (nom des membres des Cortes sous Franco) franquistes qui gagnaient ainsi l'impunité pour eux et les leurs, dans un pays où des dizaines de milliers de cadavres gisaient encore dans des fosses communes, où des fortunes s'étaient faites grâce à la répression de «l'ennemi », et où les forces de sécurité tuaient et torturaient encore. La possibilité de faire une seconde carrière dans des partis démocratiques (l'Alliance populaire, Alianza popular, et surtout l'Union du centre démocratique, Unión de centro democráico, UCD) auxquels le système électoral garantissait la victoire, ainsi que la continuité administrative (notamment dans la police et la justice) offraient quand même des garanties. L'assemblée élue dans le cadre de la réforme en juin 1977 put ainsi élaborer une Constitution démocratique pour la monarchie constitutionnelle, en vigueur à partir de janvier 1978. Le gouvernement d'Union du centre démocratique de Suárez, ayant gagné une relative paix sociale grâce aux Pactes de la Moncloa, put commencer à préparer la candidature de l'Espagne à l'entrée dans la CEE, qui allait de pair avec sa présence dans l'OTAN².

(I) Joan Garcés, Soberanos e intervenidos : estrategias globales, americanos y españoles, Madrid, Siglo XXI, 2008, $4^{\mathrm{e}}$ éd. aug. 2012 ; Pilar Ortuño Anaya, Los socialistas europeos y la transición española, Madrid, Marcial Pons, 2005 ; Bruno Vargas, «Las relaciones entre el PSOE y la Fundación Friedrich-Ebert durante el franquismo (1967-1970) », Hispania Nova, 4, 2004 ; Antonio Muñoz Sánchez, «La Fundación Ebert y el socialismo español de la dictadura a la democracia », Cuadernos de historia contemporánea, 29, 2007, p. 257-278.

(2) Les Pactes de la Moncloa furent un accord général signé en octobre 1977 entre le gouvernement, les principaux partis politiques légalisés et présents à l'assemblée élue peu auparavant, et les deux centrales syndicales UGT et Commissions
Un tel récit de la transition, devenu possible aujourd'hui grâce à la révision récente de cette histoire par des travaux eux-mêmes liés à une conjoncture de crise politique des institutions monarchiques, était impossible au cours des années $1970^{3}$. La démocratie ne pouvait pas naître d'une autoréforme de la dictature sans que personne ne soit inquiété pour sa participation à un régime des plus sanguinaires qui fut. Il fallait que la démocratie et la paix naquissent de la réconciliation entre Espagnols, entre ces deux Espagne en guerre. Cette réconciliation intervenait enfin, plusieurs décennies après une guerre fratricide, qui, quelles que fussent les raisons bonnes ou mauvaises des combattants, était une folie ayant divisé trop longtemps le pays. Ce conte à la fin heureuse s'appuyait sur l'évolution des stratégies politiques des principaux acteurs politiques et institutionnels : face à un régime dont la stratégie de légitimation passait d'abord par la victoire, le Parti socialiste, dès la fin des années 1940, puis le Parti communiste, au début de la décennie suivante, se firent les porte-parole de la « réconciliation $\gg$ face au régime de guerre de Franco. Cependant, à partir de 1964, celui-ci amorça une transition vers un système de légitimation basé sur l'idée de paix et de progrès économique; il célébra cette année-là les « vingtcinq ans de paix » garantis par le Caudillo à son pays et se fit le nouveau champion de la réconciliation face à une opposition antifranquiste taxée de dangereuse revancharde. L'alternative

ouvrières. L'accord principal portait sur la modération des revendications sociales et l'appui à une politique «d'assainissement » économique, d'esprit déflationniste, pour «stabiliser la transition $\gg$.

(3) Ferran Gallego, El mito de la transición : la crisis del franquismo y los orígenes de la democracia (1973-1977), Barcelone, Crítica, 2008 ; J. Garcés, Soberanos e intervenidos..., op. cit. Sur la dynamique interne de la dictature franquiste dans les dernières années de son existence, voir aussi le livre indispensable de Carme Molinero et Pere Ysás, Anatomía del franquismo : de la supervivencia a la agonía (1945-1977), Barcelone, Planeta, 2008. 
à la réconciliation était en effet une nouvelle guerre, menace que les propagandistes franquistes n'eurent de cesse d'agiter à chaque moment de tension politique, et en particulier pendant la transition.

La transition avait besoin du mythe de la réconciliation, qui lui-même reposait sur le mythe des deux Espagne. Le tout s'incarnait dans un adjectif, « fratricide », qui dépolitisait profondément le conflit, en ôtant toute valeur ou signification présente aux expressions politiques des sujets du passé : les raisons de ces derniers, discours surannés, pouvaient être expliquées mais pas comprises. Même si l'étiologie du conflit mobilise des motifs sociaux, religieux, politiques et culturels, l'affirmation de son caractère fratricide rend ces motifs secondaires face à l'essentiel : le frère a tué le frère. Ce récit historique met donc en avant son « impartialité », tandis que «l'esprit partisan » est le mal. Ce qui compte est alors l'image de la discipline historique parmi le public et les décideurs politiques, l'image de l'objectivité et de la science, seules susceptibles de l'emporter sur les passions, passions qui devaient être expulsées de l'arène politique où seule devait compter la volonté partagée de réconciliation.

Le processus politique en cours réserva à la guerre civile et à ceux qui en écrivaient l'histoire un lieu particulier. Un usage du passé fut décidé par consensus entre les principaux partis : il fallait préserver l'arène politique d'une invasion du passé qui, ouvrant de «vieilles blessures », risquait de ruiner les efforts des dirigeants responsables qui avaient compris la valeur de la paix, de la pitié et du pardon ${ }^{1}$. On pouvait donner libre cours à l'histoire de la guerre, mais si processus carthartique il devait y avoir, il était tout orienté vers la possibilité

(I) « Paix, pitié et pardon » était le titre du discours prononcé le 18 juillet 1938 par Manuel Azaña, président de la République espagnole, discours orienté vers la réconciliation avec l'ennemi pour faciliter une médiation internationale. de célébrer la fraternité retrouvée : en un mot, il fallait tourner la page ${ }^{2}$. Il s'agissait de ne pas mettre en danger la convivencia pacífica, qu'on pourrait traduire littéralement par le «vivre ensemble pacifique », et occuper la position suprême dans l'échelle de la morale publique au fondement (actuel et passé) du régime politique né en 1978. Tout en bas de cette échelle, on trouvait la «violence politique», associée à l'événement 1936, mais aussi mobilisée comme frontière de l'espace démocratique, par la condamnation du terrorisme, en particulier celui de Pays basque et liberté (Euskadi ta askatasuna, ETA) ${ }^{3}$. Tout en haut de l'échelle morale et historique, on trouve la transition elle-même, réputée pacifique, projetée comme un modèle par les dirigeants politiques et les principaux médias, associée à un esprit d'union nationale qui doit s'incarner périodiquement dans des pactes entre les deux grands partis de gouvernement ${ }^{4}$. Entre les deux, la peur d'une répétition de la guerre était omniprésente : «la peur est le troisième personnage de notre réalité politique», disait Manuel Vázquez Montalbán ${ }^{5}$. Le triomphe de l'expression «guerre civile» à partir des années 1960 était lié à la mise en avant de son dépassement, à la mise en marche d'une nouvelle

(2) Paloma Aguilar a montré depuis plusieurs années que le « pacte de silence » dénoncé par les associations mémorielles à propos des politiques de mémoire de la transition vis-à-vis de la guerre a été en réalité un pacte de séparation entre espace public/politique et espace de la narration du passé. Combattant cette idée de « pacte de silence », l'historien Santos Juliá a souligné la très grande quantité de la production historique de la période 1978-1986.

(3) La condamnation du terrorisme est ainsi devenue, à partir des années 1980 et surtout des années 1990, le marqueur d'appartenance à l'espace public légitime.

(4) Cela est démenti par les travaux de Sophie Baby et de Pau Casanellas : Sophy Baby, Le Mythe de la transition pacifique : violence et politique en Espagne (1975-1982), Madrid, Casa de Velázquez, 2012 ; Pau Casanellas, Morir matando: el franquismo ante la práctica armada (1968-1977), Madrid, Libros de la Catarata, 2014.

(5) Manuel Vázquez Montalbán, «Violencia latente », Triunfo, 765, 24 septembre 1977. 
Espagne ${ }^{1}$. Cette idéologie eut sa version franquiste (qu'on a appelé le desarrollismo, littéralement « développementisme ») et sa version transitionnelle.

\section{Des historiens et des archives}

Les conditions culturelles de production de l'histoire de la guerre telles que nous les avons décrites furent renforcées par l'image que la discipline avait dans le public et parmi les faiseurs d'opinion. L'histoire était perçue comme une science objective, qui faisait parler les archives. L'objectivité avait valeur «d'orthodoxie », pour reprendre la formule de l'historien Pablo Sánchez León, auteur d'une première approche de cette historiographie en termes de génération intellectuelle ${ }^{2}$. Rappelant que les historiens peuvent être pris comme des représentants de leur époque, il ajoutait :

«Leur fonction sociale consiste à fournir aux identités collectives une interprétation ordonnée du passé historique capable de rendre intelligible

(I) L'expression «guerre civile» est presque systématiquement rejetée par les discours de mobilisation pendant les guerres internes elles-mêmes en ce qu'il revient à conférer aux ennemis la dignité de «nationaux » alors qu'il s'agit précisément de les expulser de la communauté imaginée que l'on est en train de (re)fonder. Sur cette question, voir Jean-Claude Caron, Frères de sang : la guerre civile en France, Paris, Champ Vallon, 2009 ; Claudio Pavone, Una gerra civile : saggio storico moralità nella Resistenza, Turin, Bollati Boringhieri, 1991 ; trad. fr., id., La Guerre civile : essai historique sur l'éthique de la résistance italienne, préf. Bernard Groz, trad. de l'it. par Jérôme Grossman, Paris, Éd. du Seuil, 2005. Sur les noms du conflit de 1936, voir François Godicheau, « Guerra civil, guerra incivil : los nombres de la guerra », in Julio Aróstegui Sánchez et François Godicheau (dir.), Guerra civil: mito y memoria, Madrid, Marcial Pons, 2006, p. 143-167 (actes du colloque «Memoria e historiografía de la guerra civil (1936-1939)», Casa de Velázquez, Madrid, 4 et 5 mars 2002); sur la notion de « guerre civile», voir François Godicheau, « La guerra civil, figura del desorden público : el concepto de guerra civil y la definición del orden público », in Jordi Canal et Eduardo González Calleja (coord.), Guerras civiles : una clave para entender la Europa de los siglos XIX y XX, Madrid, Casa de Velázquez, 2012 , p. $75-88$.

(2) Clásicos de historia social en España : una selección crítica, Valence, Historia social, 2000. aux membres de la communauté leur origine et leur évolution jusqu'au présent ${ }^{3}$.»

Il formulait alors un jugement qui explique bien le fonctionnement de l'historiographie de la guerre d'Espagne et ses rapports avec les courants d'opinion sur le passé :

« L'historiographie sur la guerre civile espagnole instituée au cours de ces trente dernières années, alors qu'elle manque des ressources épistémologiques suffisantes pour atteindre son objectif de légitimation sociale, est considérée par convention comme impartiale et objective. Les interprétations qu'elle parvient à établir par consensus sont prises comme des verdicts sans appel et instituées comme des vérités. Quant aux historiens, ils ont été érigés en interprètes exclusifs de la mémoire collective ${ }^{4}$. »

Cette situation n'est sans doute pas spécifique à l'Espagne, mais elle prit un tour particulier pendant la transition.

La polémique de 1977 autour de l'histoire de Guernica est très éclairante à ce sujet. La querelle ne portait pas sur les chiffres de victimes du bombardement par la légion Condor, mais sur la réalité même du bombardement: quarante ans après les faits, la version officielle était toujours celle de l'état-major franquiste, selon lequel la destruction de la ville était due à un incendie volontaire des soldats républicains fuyant devant les troupes nacionales. Lors du quarantième anniversaire du massacre, une assemblée réunissant témoins et historiens envoya un communiqué au gouvernement pour demander, d'une part, le changement de la version officielle, franquiste, des faits, et, d'autre part, l'ouverture immédiate

(3) Pablo Sánchez León, « La objetividad como ortodoxia : los historiadores y el conocimiento de la guerra civil española », in J. Aróstegui Sánchez et F. Godicheau (dir.), Guerra civil..., op. cit., p. 75-106.

(4) Ibid., p. 117. 
des archives de la guerre, en particulier celles conservées à Salamanque. La distinction des deux termes de la revendication renvoyait au fait que l'abandon du récit franquiste ne demandait pas de nouvelles preuves mais était une question politique. Était d'abord en jeu la caractérisation du mythe franquiste comme mythe, et ensuite sa déconstruction, acte politique auquel pouvaient participer des historiens. Au début de l'année 1978, les archives restaient fermées au public. Le tout nouveau gouvernement de l'Union du centre démocratique, présidé par Adolfo Suárez, permit à un membre d'une commission d'historiens désignée officiellement d'accéder à la documentation. Le journal El País expliqua la démarche du ministère de la Culture du moment :

« Le gouvernement espagnol n'acceptera de réviser la version officielle sur le bombardement qu'à partir du moment où les recherches de groupes d'historiens espagnols et allemands auront démontré de manière documentée et scientifique ce qui s'est passé à Guernica en avril $1937^{1}$. »

Pour éviter le sentiment d'arbitraire qu'aurait entrâné l'adoption de la version antifranquiste, le gouvernement se réfugiait derrière « l'objectivité » et « les archives ». Ainsi la version franquiste fut remplacée par un nouveau récit qui ne modifiait pas en substance les faits connus, mais qui s'appuyait sur des documents d'archives et non plus sur des témoignages. À aucun moment la vieille version officielle ne fut publiquement condamnée.

Un an après, en mai 1979, les fonds du Service documentaire du régime entreposés à Salamanque, et dont il avait été fait depuis la guerre un usage policier, devinrent une annexe des Archives nationales. Il s'agit du principal fonds d'archives sur le conflit pour l'étude du

(I) Cité dans Jesús Izquierdo Martín et Pablo Sánchez León, La guerra que nos han contado : 1936 y nosotros, Madrid, Alianza, 2006, p. 54 sq. camp républicain, et sans doute le plus riche. Il est le résultat de la collecte systématique, par les armées victorieuses des papiers des organisations politiques et syndicales ainsi que des institutions officielles de la République. Or cet ensemble de fonds n'a commencé à faire l'objet d'un catalogage précisé qu'à partir de $2007^{2}$. À la fin des années 1990, le chercheur avait à sa disposition plusieurs volumes de tapuscrits qui décrivaient de manière très succincte et souvent complètement erronée le contenu des milliers de cartons : ces volumes avaient été réalisés par la garde civile, au moment où, à la fin des années 1970, ces archives avaient commencé à être ouvertes à la consultation. Dans le volume sur la série « Político social Barcelona », la plus importante avec celle de Madrid, les premières dizaines de cartons faisaient l'objet d'une description assez vague d'environs dix à quinze lignes par carton, en tenant compte du fait que se mêlaient à l'intérieur de ceux-ci des documents de toute provenance, type, date, sans le moindre ordre ni logique. Cependant, au fur et à mesure que l'on avançait dans ce volume qui ne comportait évidemment aucun index, les descriptions se limitaient à deux ou trois lignes par carton, ou à une demi-ligne, traduisant l'épuisement et l'ennui des gardes affectés à cette tâche pour laquelle ils n'avaient

(2) Aujourd'hui, les « archives générales de la guerre civile » sont devenues Centro documental de la memoria histórica, et accueillent de nombreuses autres collections, même si, en volume, la documentation originelle continue de primer. On y trouve des dépôts privés, de personnes, de familles et des diverses « associations de récupération de la mémoire historique $\gg$. Un certain nombre de petits fonds ont été catalogués et certains sont mêmes consultables en version numérique depuis Internet. Le ministère de l'Éducation et de la Culture espagnol a en effet développé un système national de recherche en ligne qui permet d'accéder aux catalogues et à de très nombreux fonds numérisés d'une douzaine de centres d'archives importants, avec des fonds volumineux, bien catalogués, accessibles par mots clés et de nombreux documents en ligne. À Salamanque, les grandes séries « Político social » de Madrid et de Barcelone ont été cataloguées en partie et sont disponibles à travers le portail des archives espagnoles Pares (http://pares.mcu.es/). 
pas été préparés. Jamais ces fonds n'avaient été triés par des archivistes et aucune trace de classement original n'était perceptible. Seul le fait que les noms propres étaient soulignés en rouge attestait de son usage policier pendant la dictature pour nourrir un fichier nominatif où les responsabilités politiques des uns et des autres figuraient, avec des erreurs monumentales. Seule la documentation liée aux organisations libertaires avait fait l'objet d'un repérage à peu près systématique par une fondation privée, militante, qui avait déposé ses propres tapuscrits identifiant les documents liasse par liasse. Cet état de la documentation constituait une aubaine fantastique pour un débutant enthousiaste disposé à ouvrir des centaines de cartons « au jugé ${ }^{1} »$.

Si l'histoire de la guerre de 1936 était si importante dans le pays, comment se faisaitil que le principal gisement documentaire, des fonds donnant accès directement aux expériences de la société en guerre, soit laissé dans cet état ? Cette situation pourrait sembler paradoxale dans la mesure où l'orthodoxie de l'objectivité reposait sur ce que les archives étaient censées dire. L'accès aux archives et l'élaboration de nouveaux faits n'ont souvent servi qu'à compléter un récit de la guerre dont les cadres furent posés à partir des années 1960 ; à le compléter, à l'affiner, à le corriger sur certain point, dans le cadre des fonctions assignées à l'histoire et à des valeurs à transmettre via la narration de ce passé.

\section{Un modèle de récit consacré}

Depuis une quinzaine d'années, de nombreux auteurs ont mobilisé à propos des récits du passé la notion de mythe, mais la plupart du

(I) Cela nous a effectivement permis de mettre au jour des phénomènes peu connus, voire inconnus, indispensables pour réinterpréter l'évolution politique de la Catalogne en guerre et celle du mouvement libertaire dans son ensemble. temps comme synonyme de récit mensonger. On peut aussi qualifier le métarécit hégémonique de la guerre fratricide de mythe, mais au sens fort, souligné par Roland Barthes ${ }^{2}$. Pour lui, ce qui importe dans le mythe, contemporain ou antique, n'est pas qu'il dise le vrai ou le faux, mais le système des valeurs transmises et reconnues comme propres par le public qu'il qualifie de « consommateur de mythes ». La difficulté vient du fait que le mythe, écrit-il, « est une parole définie par son intention beaucoup plus que par sa lettre $\gg$. Pourtant, ajoutet-il, «l'intention y est en quelque sorte figée, purifiée, éternisée, absentée par la lettre » : on s'instruit en lisant des livres sur la guerre mais l'interprétation générale est déjà donnée et même sue. L'historien serait presque devenu un antiquaire si l'émergence d'un révisionnisme négationniste et la mise en cause par les mouvements mémoriels ne l'avaient poussé à changer d'attitude, mais guère de langage ${ }^{3}$.

On retrouve dans le mythe barthien un certain nombre de caractéristiques générales de l'historiographie de la guerre d'Espagne, lesquelles découlent d'un corollaire pointé par le même Barthes: le mythe est, selon lui, une « parole dépolitisée ». Loin du silence, il raconte, et le simple fait de raconter dans un cadre coupé des questions politiques (le fameux pacte de la transition) permet au mythe de s'incarner et d'atteindre son efficacité. « La leçon de l'Histoire » prend appui sur une multitude de descriptions qui reproduisent souvent le même schéma narratif. Lors d'un colloque organisé à la Casa de Velázquez à Madrid en 2002 sur le poids des questions de mémoire dans l'écriture de l'histoire de la guerre ${ }^{4}$, le

(2) Roland Barthes, Mythologies, Paris, Éd. du Seuil, 1957, 1970, p. 204.

(3) Ibid., p. 197.

(4) «Memoria e historiografía de la guerra civil (19361939) », Madrid, Casa de Velázquez, 4 et 5 mars 2002. Voir J. Aróstegui Sánchez et F. Godicheau (dir.), Guerra civil..., op. cit. 
directeur de l'Institut d'histoire du temps présent (IHTP), présent avec quelques collègues français, fut très surpris d'entendre un professeur de l'Université de Salamanque se féliciter du caractère très complet de la connaissance historique sur la guerre d'Espagne et signaler qu'il ne manquait plus que le couronnement de ce grand'œuvre, « la synthèse définitive » sur le sujet. Certes, cette conception cumulative du savoir historique n'est pas propre à l'histoire de la guerre de 1936 et il faudrait la resituer dans une réflexion plus large sur une génération d'historiens qui, après quatre décennies de dictature, a dû défricher une histoire contemporaine souvent vierge d'études scientifiques. Toutefois, le fait que bien souvent, ce qui préside au choix par les étudiants espagnols d'un sujet de mémoire, puis de thèse de doctorat, est la recherche d'une lacune dans la connaissance, d'un thème non encore traité, donne des résultats particuliers dans le cadre de l'événement qui nous occupe, où des centaines d'études se concentrent sur à peine trois ans: la reproduction du même récit ou du moins du même patron narratif, comme s'il s'agissait d'illustrer encore et encore une histoire par ailleurs «bien connue ${ }^{1} »$.

Depuis plusieurs décennies, il existe un consensus sur l'ordre d'importance des « grandes questions $\gg$ sur le conflit : pourquoi les uns ont perdu et les autres gagné, les « répressions » s'équivalent-elles, qui est responsable de la guerre et de son dénouement, quel est le poids des «alliés» étrangers des uns et des autres. En réalité, la plupart des nouveaux livres sur l'histoire de la guerre se coulent dans la structure posée pendant les années 1960 par un

(I) Plus d'éléments sur cet aspect dans François Godicheau, « "L'histoire objective" de la guerre civile et la mythologie de la transition », in Corrado Danielle et Alary Viviane (coord.), La Guerre d'Espagne en héritage : entre mémoire et oubli (de 1975 à nos jours), Clermont-Ferrand, Presses universitaires Blaise-Pascal, 2007, p. 69-96. groupe d'éminents historiens étrangers (non espagnols) ${ }^{2}$. Cette historiographie étrangère proposait une première narration qui cherchait l'objectivité, c'est-à-dire à échapper aux propagandes des deux camps, et tout spécialement à celle des vainqueurs, qui depuis 1939 s'était imposée de façon implacable. Hugh Thomas rappelait dans la préface à l'édition de 1976 de son ouvrage La guerra civil española que le régime diffusait sa vision du conflit à travers une série de fascicules, les temas españoles, centrés sur des événements héroïques ou tragiques ayant popularisé efficacement un kaleidoscope de la guerre fait d'images de propagande.

Lobjectif premier était donc d'offrir un récit événementiel complet, ce que fit l'historien anglais. Il ne faut pas négliger la force de cette première composition d'ensemble qui organise la compréhension de la guerre. Ellemême était très dépendante des thèmes que les propagandes des diverses parties (non seulement chaque camp, mais aussi les organisations politiques à l'intérieur de ceux-ci) avaient agités dans leurs journaux et leurs affiches. L'entreprise consistant à examiner « les faits » tout d'abord «sans passion et sans idées préconçues $^{3} \gg$ partait de la construction de ces mêmes faits, effectuée pendant un conflit qui avait été saturé de discours de justification de soi et de mise en cause de l'ennemi.

Le fait que le régime franquiste et les partis de l'exil ou de la résistance intérieure continuaient à utiliser, au moins en partie, les rhétoriques de la guerre ou au moins les élements d'identification de l'ennemi alourdit le poids de cette construction des sujets et de cette sélection des thèmes historiques, datée de 1936-1939, sur le présent de l'écriture historique. Les narrations proposées par les historiens étrangers à partir

(2) Voir la contribution d'Élodie Richard et Charlotte Vorms dans ce numéro.

(3) Hugh Thomas, La guerra civil española, París, Ruedo ibérico, 1976, préf. p. vi. 
de ce matériau et orientées vers une objectivité pacificatrice atteignirent globalement leur objectif, celui de l'établissement d'une histoire commune pouvant être adoptée de part et d'autre comme vraie et utile. On peut ainsi dire que le modèle d'interprétation dominant déroule une histoire de la guerre dont les termes sont largement hérités de l'époque de la guerre elle-même. Il s'agit d'une histoire politique de facture très classique où dominent des dirigeants politiques et des acteurs collectifs réifiés et identifiés à partir des sigles des organisations politiques et syndicales ou des grands découpages sociaux (bourgeoisie, aristocratie, petite bourgeoisie, ouvriers, paysans, paysans sans terre, clergé, militaires). Si, pendant les années 1960, les personnages éminents dominaient dans le récit, l'histoire sociale donna une place aux groupes sociaux, conjugués aux différents sigles. Les changements sociaux intervenus au cours de la guerre n'ont en revanche pratiquement pas été étudiés et les organisations politiques, quand elles font l'objet de monographies, ne sont considérées qu'à partir des divisions qui agitaient leurs cercles dirigeants.

Les études consacrées aux acteurs anonymes ou à des localités ou des cantons (l'histoire locale représentant une grande part de la production de livres sur la guerre) sont, à de très rares exceptions près, des déclinaisons du métarécit de la guerre et de sa structure chronologique, avec une série de «passages obligés » correspondant aux formes locales des grands événements de la guerre. Tout commence par un rappel de l'histoire de la Seconde République proclamée en 1931, censée être l'antichambre de la guerre : le récit mêle une mention des «contradictions sociales » de l'Espagne d'alors, évoquant les paysans sans terre, la réforme agraire, caractérisée comme courageuse mais insuffisante, timide parfois, sabotée et incomprise; évoquant aussi les conséquences de la crise économique mondiale dans le pays, la particularité d'un mouvement ouvrier à forte présence anarchiste et le maximalisme de ce courant dont les tentatives insurrectionnelles «fragilisent» le régime. On trouve aussi une mention de l'Espagne traditionnelle catholique qui refuse le libéralisme. La République devient un régime incapable de résoudre ces contradictions dans un contexte international tendu. Les législatures se succèdent dans une montée aux extrêmes qui débouche sur un printemps 1936 de tous les dangers.

Le coup d'État militaire des 17-18 juillet 1936 intervient alors. Un tour du pays est effectué, détaillant la manière dont l'épreuve tourne à l'avantage du gouvernement ou des rebelles. Deux thèmes sont mis en avant : d'une part la description de la révolution dans le camp républicain et de la faiblesse du gouvernement, de l'autre la première structuration du pouvoir rebelle et l'arrivée de Franco à la tête de la junte. Vient alors l'histoire de la « répression » dans un camp et dans l'autre (l'histoire de la répression formant un sous-genre à elle seule), avec la description de ses formes (d'abord sommaires puis de plus en plus judiciaires) et quelques épisodes incontournables car très utilisés par les propagandes des deux camps pendant des années (il faut parler du massacre des prisonniers rebelles à Paracuellos del Jarama, des massacres perpétrés par les militaires à Badajoz, en Extrémadure, il faut parler de la mort du poète García Lorca et du sort du dirigeant de la Phalange José Antonio Primo de Rivera). Les alignements internationaux doivent ensuite être évoqués, Hitler et Mussolini du côté de Franco, non-intervention de la France, de l'Angleterre, et aide soviétique. On passe ainsi naturellement à la bataille de Madrid et à l'intervention des Brigades internationales.

L'année 1937 peut commencer par un récit des divisions politiques républicaines 
(la montée du Parti communiste, son affrontement avec Francisco Largo Caballero, luimême opposé à Indalecio Prieto au sein du Parti socialiste ouvrier, la question de la militarisation des milices de volontaires) parallèle au renforcement du pouvoir de Franco, en laissant une place, si cela n'a pas été fait avant, à l'examen de la position de l'Église. En intercalant la prise de Malaga par les franquistes en février, on arrive ainsi à la crise du printemps 1937 de part et d'autre du front (affrontements armés à Barcelone début mai entre révolutionnaires et gouvernement catalan, mise au pas de la Phalange en avril par le « généralissime »). On peut alors reprendre l'histoire des batailles, sur le front de Madrid, puis celui d'Aragon (avec un passage sur le collectivisme anarchiste et sa dissolution par l'Armée populaire commandée par le communiste Enrique Lister). On passe ensuite au front nord, du Pays basque aux Asturies, en traitant du bombardement de Guernica, et on finit le récit des défaites militaires républicaines par l'évocation des conditions difficiles d'un deuxième hiver dans le camp républicain.

L'année 1938 est beaucoup plus vite racontée : la politique intérieure au camp républicain y disparaît presque, sauf pour les rapports entre le chef du gouvernement, le socialiste Juan Negrín, et les communistes. On peut en profiter pour décrire un peu la vie dans la nouvelle Espagne franquiste, de l'autre côté du front. Les batailles s'enchaînent jusqu'à celle de l'Ebre pendant l'été, ce bref sursaut républicain, et un développement peut être consacré de nouveau à la lutte du gouvernement républicain pour briser le cercle de la non-intervention, jusqu'à ce qu'à l'automne tout s'écroule. La fin du conflit demande un récit sur la retirada et les masses de réfugiés qui affluent en France, puis un retour aux luttes internes à ce qui reste du camp républicain après la chute de la Catalogne, pour finir sur le coup d'État de mars 1939 à Madrid et au point final de la guerre. Depuis le milieu des années 1990, un dernier chapitre sur la répression orchestrée par les vainqueurs à partir de 1939 vient interroger le découpage chronologique canonique d'une guerre sur trois ans.

Tous les thèmes évoqués ici ont été saturés de discours partisans contradictoires, puis traités avec une recherche de neutralité par les grandes synthèses des années 1960, enfin précisés, corrigés, ciselés par des quantités considérables de travaux empiriques. Tous sont pratiquement obligatoires dans une histoire générale du conflit: chacun des nombreux personnages politiques doit avoir sinon un portrait du moins être rapporté à ses actions les plus connues, et beaucoup de sujets charrient des argumentaires attendus.

\section{Une histoire patrimoine}

Le récit que nous venons de décrire fonctionne comme un patrimoine politique du régime né en 1978. À ce titre, il peut être revendiqué, décliné en différentes versions, mais il peut être aussi une source de problèmes en raison de l'évolution politique de l'Espagne et de la crise, latente depuis la fin des années 1990 et ouverte depuis quatre ans, de ce même régime ${ }^{1}$.

Le colloque madrilène de 2002 déjà évoqué était la première réflexion collective sur la place du passé de guerre civile dans la société espagnole. Il avait été organisé pour traiter à la fois du poids des politiques de mémoire sur le travail des historiens et de la place de celuici dans la société. Le métarécit élaboré depuis la transition avait déjà commencé à être mis en question par des associations et par ce qui

(I) Les historiens étrangers n'échappent en général pas à la force de ce métarécit. Ils s'inscrivent le plus souvent dans la tradition des « hispanistes » des années 1960. La célébration de la transition empêche toute approche critique sur l'interprétation de la guerre comme fratricide, sauf bien sûr dans les traditions d'extrême gauche d'histoire militante. 
allait devenir le «mouvement de la mémoire » évoqué plus largement dans ce numéro de Vingtième Siècle. Un livre, La memoria insumisa, écrit par un ancien dirigeant et par un avocat des Commissions ouvrières, le syndicat lié au Parti communiste, protestait contre une des conséquences du mythe de la réconciliation : celui-ci faisait du franquisme une dictature bon enfant et éclairée, qui aurait fait sortir l'Espagne du sous-développement et l'aurait finalement propulsée dans la modernité ${ }^{1}$. Sur le plan strictement historiographique, des études sur la dictature comme fascisme espagnol et sur son système répressif se multiplièrent à partir de la fin des années $1990^{2}$. Par ailleurs, un historien important spécialiste de la question de l'épuration pendant la guerre, Alberto Reig Tapia, publiait en 1999 Memoria de la guerra civil: los mitos de la tribu, où il s'attaquait aux récits mensongers nés de propagande franquiste et profondément ancrés dans le savoir populaire sur l'époque ${ }^{3}$. La réflexion sur les rapports de la société espagnole à la guerre de 1936 était, nous le découvrîmes en 2002, un territoire immense, qui a depuis été cultivé par des historiens, des anthropologues comme Francisco Ferrándiz, mais assez peu par des sociologues de la mémoire ou par des politistes. La question des conditions de production de l'historiographie et des usages sociaux des différents récits n'a en particulier pas fait l'objet d'une réflexion systématique.

Sans prétendre mener ici ce travail nécessairement collectif et de longue haleine, on peut simplement reprendre cette idée du récit comme patrimoine pour évoquer quelques

(I) Nicolás Sartorius et Javier Alfaya, La memoria insumisa : sobre la dictadura de Franco, Madrid, Espasa Calpe, 1999.

(2) Nous faisons référence aux travaux du Groupe de recherches sur l'Espagne franquiste, le GREF, de Barcelone, et à d'autres ouvrages importants comme ceux de Conxita Mir Curcó, Angela Cenarro et Javier Rodrigo.

(3) Alberto Reig Tapia, Memoria de la guerra civil : los mitos de la tribu, Madrid, Alianza, 1999. pistes, et notamment les mises en cause du métarécit hégémonique et la manière dont celui-ci s'adapte au gré de l'évolution politique. Si ce récit est un patrimoine politique du régime de 1978, il ne répond pas également à toutes les demandes adressées à l'historiographie, à la narration du passé. Le mouvement de revendication mémorielle né à la fin des années 1990 et qui, depuis, a pris une ampleur considérable et a porté le débat dans l'arène politique est sans doute lié à la difficulté pour de nombreux individus, familles et courants politiques à s'y reconnaître. Comme l'ont montré Pablo Sánchez León et Jesús Izquierdo Martín dans leur livre 1936 et nous : la guerre qu'on nous a racontée, la principale source de narrations sur le passé est longtemps restée et reste sans doute encore le récit au sein de la famille ${ }^{4}$. Les multiples histoires de famille, transmettant des valeurs propres à celle-ci, ont été racontées en parallèle au métarécit que l'on trouvait dans les livres. Dans la plupart des cas, il était difficile de faire coïncider les unes et les autres, car le grand récit historique de la guerre ne donnait à voir que des sujets collectifs, dont l'engagement idéologique était complètement naturalisé, et des figures éminentes dont le défilé ne permettait pas d'éclairer les mésaventures des parents et des grands-parents. S'il était communément admis que l'histoire du malheur familial illustrait le malheur national, dans le détail, les choses étaient plus compliquées. Pour les personnes dont les familles appartenaient aux diverses traditions militantes de l'antifranquisme, des récits alternatifs existaient, en particulier pour le mouvement libertaire, quasiment disparu du paysage politique, même si ces livres étaient souvent anciens et ne reflétaient que très partiellement les

(4) J. Izquierdo Martín et P. Sánchez León, La guerra que nos han contado..., op. cit. 
souvenirs d'expériences transmis par les militants ${ }^{1}$. Cependant, dans l'ensemble, les traditions familiales du militantisme antifranquiste étaient exclues du récit dominant.

La prégnance du cadre familial et, par conséquent, l'importance du décalage avec le discours public sur le passé étaient d'autant plus notables que la transmission par l'école ne se faisait pas, ou peu. Pendant de longues années de la dictature, l'enseignement de l'histoire ou de la matière appelée « formation de l'esprit national » était centré sur le récit de la « croisade », car le régime fondait sa légitimité sur la victoire de 1939. Même si on ne dispose que d'une étude sur les manuels d'histoire de la dictature, on sait que ceux-ci évoluèrent en fonction de la stratégie de légitimation du régime $^{2}$. En 1957 par exemple, si l'on examine le nom du conflit, on trouve plus souvent « guerre civile» et, en 1967, celui de « croisade » disparaît, ainsi que le récit des hauts faits des vainqueurs, comme l'épisode de l'Alcazar de Tolède. «La grande tragédie de la lutte civile » est évoquée mais peu d'éléments sont en revanche donnés sur les causes de la guerre, et quand c'est le cas, les manuels parlent essentiellement de rétablissement de l'ordre. Dans les manuels de 1975, les mois du gouvernement de Front populaire entre février et juillet 1936 représentent toujours autant le désordre,

(I) Nous pensons, par exemple, au livre classique de José Peirats, La CNT en la revolución española (Toulouse, CNT, 1971) commande de la direction de la Confédération nationale du travail (Confederación nacional del trabajo, CNT) en exil. Cet ouvrage reflétait le point de vue politique de celle-ci sur le déroulement des événements. Il fournissait un récit officiel, qui certes transmettait les valeurs du groupe mais qui excluait aussi des pans entiers de cette histoire, en particulier les terribles divisions ayant agité les rangs libertaires et qui constituaient une partie des souvenirs les plus forts, toujours tus, de nombreux militants.

(2) José Antonio Álvarez Osés, Ignacio Cal Freire, Juan Haro Sabater et María del Carmen González Muñoz, La guerra que aprendieron los españoles : república y guerra civil en los textos de bachillerato (1938-1983), Madrid, La Catarata, 2000. C'est de ce livre que nous tirons les éléments de ce paragraphe. mais la faute n'en revient plus seulement aux « extrémistes de gauche »; on parle de «pistoleros de l'un et l'autre camp » et on parle même de la violence et du désordre comme prétextes pour le déclenchement des hostilités par les classes conservatrices par peur du bolchévisme. Cependant, la plupart ne s'engagent pas vraiment et se contentent de présenter ces mois comme un crescendo révolutionnaire. Il est à noter que cette année-là, le livre le plus cité est celui de Hugh Thomas ${ }^{3}$. Enfin, il apparaît qu'avec la question du nombre de victimes de part et d'autre, le thème, décisif, de l'aide étrangère aux deux camps est le plus manipulé.

Au-delà des manuels scolaires, il semble, d'après le livre de Pablo Sánchez León et Jesús Izquierdo Martín, que l'enseignement réel de l'histoire de la guerre dans le temps scolaire a été très rare pour toute une génération, âgée aujourd'hui d'entre trente et cinquante-cinq ans. L'histoire de cette période n'a ainsi pas été l'objet d'un travail d'intégration nationale et de formation de consciences citoyennes susceptibles d'incorporer ce passé à une identité politique au présent ${ }^{4}$.

C'est dans la multiplication des livres d'histoire locale que la fonction de patrimoine politique du métarécit de la guerre apparaît le plus nettement, car on trouve la matière humaine (les aspects individuels de l'histoire, les souvenirs) intégrés au patron général. L'étude de la production catalane, très dense (tous les cantons ont leur histoire et dans certains, plusieurs villages ont la leur propre) peut l'illustrer $^{5}$. Ces ouvrages sont le plus souvent des

(3) Puis, à égalité, les livres de l'historien franquiste Ricardo de la Cierva, du grand historien antifranquiste Manuel Tuñón de Lara et de l'Anglais Gabriel Jackson.

(4) Sur la notion de «travail de la mémoire », voir MarieClaire Lavabre, « Sociologia de la memoria y acontecimientos traumaticos », in J. Aróstegui Sánchez et F. Godicheau (dir.), Guerra civil..., op. cit., p. 31-55.

(5) François Godicheau, «"L'histoire objective" de la guerre civile et la mythologie de la transition », in Corrado Danielle et Alary Viviane (coord.), La Guerre d'Espagne en 
chroniques qui déclinent en termes locaux le grand récit national : ils suivent des étapes « obligées », réduisant le métarécit à sa plus simple expression. Chaque chapitre établit un véritable dialogue entre les souvenirs individuels ou familiaux des lecteurs et les renseignements inconnus qui vont satisfaire sa curiosité et enrichir sa «mémoire de la guerre ». Ce dialogue passe par le texte, mais aussi par les photographies souvent très nombreuses, qui jouent le rôle de preuves, comme les listes de morts, de miliciens ou de prisonniers, qui sont chargées de transformer la rumeur en fait avéré. Ces livres ont ainsi un rôle de reconstitution qui intéresse les habitants, lesquels peuvent y retrouver des proches ou des photographies d'eux-mêmes : ils permettent d'incarner la continuité des vies, celle des individus et celle du village. En recréant la communauté d'antan, celle-ci même qui était déchirée, partagée en son milieu par la guerre, ils figurent et célèbrent sa cohésion finale, la reconstitution de la communauté dans le présent. Introductions et préfaces, qui formulent le vœu que cette horreur et cette misère ne se reproduisent plus, achèvent de faire de ces livres des espèces de totems où la guerre fratricide est conjurée et vaincue par la convivencia pacífica.

\section{Un patrimoine disputé}

Utilisé pour la réassurance de la communauté pacifiée de la démocratie enfin revenue, ce patrimoine historique de la guerre civile peut être aussi disputé. Un groupe d'historiens autoproclamés, avec en son cœur un écrivain nommé Pío Moa, a ainsi entrepris depuis la fin des années 1990 une « révision » de l'histoire de la guerre. Liés à une sorte de think tank idéologique qui représente la fraction la plus conservatrice du Parti populaire,

héritage : entre mémoire et oubli (de 1975 à nos jours), ClermontFerrand, Presses universitaires Blaise-Pascal, 2007, p. 69-96. ces auteurs ne cachent pas leurs sympathies pour le franquisme, mais jouent habilement avec les limites du récit hégémonique et avec l'idéal d'une histoire objective et antipartisane $^{1}$. Dans son ouvrage vendu à des centaines de milliers d'exemplaires, Los mitos de la guerra civil, Pío Moa reprend, en leur donnant un lustre «savant », une bonne partie des éléments de la propagande de la dictature à propos de 1936, et offre à ses lecteurs une narration qui plaît, car elle mêle des éléments d'une histoire «connue » (transmise en réalité par l'école franquiste ou des traditions familiales nostalgiques) à des données reprises dans des études historiques sérieuses.

La stratégie de cet auteur consiste, tout en soutenant des falsifications qui font s'étrangler d'indignation tous les historiens de profession, à accuser ceux-ci d'être les représentants d'une idéologie de gauche mystificatrice et à prétendre parler sans tabous, pour exprimer

(I) Pío Moa est la figure de proue d'un groupe « historiographique » qui comprend César Vidal (auteur de plus de cent livres sur des sujets très variés), Luis Togores, Julios Ruiz, Angel David Martín Rubio. L'offensive idéologique qu'il incarne, décrite par Alberto Reig Tapia dans Anti-Moa: la subversión neofranquista de la bistoria de España (Barcelone, Ediciones B, 2006), s'est appuyée sur des personnages importants des médias conservateurs, parmi lesquels on peut citer Jaime Campany, décédé en 2005, ancien directeur de Arriba!, organe central de la Phalange, collaborateur du quotidien conservateur $A B C$ et de la Cadena de ondas populares españolas (COPE), la radio de la Conférence épiscopale espagnole ; Miguel Ángel Rodríguez, directeur de communication du Parti populaire de 1988 à 1996, puis porte-parole du gouvernement Aznar, collaborateur du quotidien conservateur La Razón, de la COPE, et de divers médias en ligne ; José María Marco, professeur à l'Université Pontificia Comillas, collaborateur de La Razón, de la COPE, collaborateur également de José María Aznar pour la rédaction de ses livres ; Federico Jiménez Losantos, présentateur à la $\mathrm{COPE}$, éditorialiste à $A B C$ puis au quotidien El Mundo, également très présent sur la chaîne de télévision Intereconomía, il est devenu directeur du groupe Libertad digital où il s'exprime dans le quotidien du même nom et sur sa radio EsRadio ; José María Zavala, collaborateur de La Razón, d'El Mundo, de la revue La Aventura de la bistoria et d'Intereconomía televisión ; Alfonso Ussia, collaborateur d'ABC puis de La Razón, de COPE et de Telecinco; et Isabel Sansebastián, collaboratrice d' $A B C$, de COPE, de Punto Radio, de Telemadrid et de Telecinco. 
finalement des opinions profranquistes devenues difficiles à soutenir publiquement, en particulier pendant les longues années où le Parti socialiste dominait incontestablement une droite n'ayant jamais vraiment renié son passé. L'histoire universitaire devient alors les «mythes » et le bricolage de Pío Moa, une vérité désintéressée et objective, une façon de « regarder les choses en face », au service, bien sûr, du « vivre ensemble pacifique »d'une démocratie moderne. Ici, l'accusation faite autrefois aux anarchistes d'avoir fragilisé le cadre légal républicain par leurs insurrections, est lancée aux socialistes. Ceux-ci seraient coupables d'avoir soutenu la grève générale insurrectionnelle de 1934 aux Asturies et donc d'avoir les premiers rompu avec le cadre légal du régime, ce qui faisait du coup d'État de juillet 1936 une réponse presque logique, sinon légitime, conformément à la version franquiste de l'histoire.

Face à cette offensive, les historiens se sont retrouvés en mauvaise posture. La plupart ont réagi par le mépris des professionnels envers les amateurs, ne s'abaissant pas à polémiquer avec des propagandistes. Cependant, ceux-ci passant pour historiens auprès du grand public, certains collègues sont malgré tout entrés dans l'arène. Utilisant une argumentation visant à rétablir les faits à partir de la documentation, sans aucun doute nécessaire dans la mesure où les falsifications de Pío Moa prennent appui sur des éléments précis déguisés sous une fausse érudition, ils n'ont pas commencé par attaquer directement le registre d'interprétation de ces publicistes réactionnaires qui se désignaient comme des révisionnistes. Le problème était que les attaques de ces propagandistes étaient construites dans le même registre que la narration hégémonique produite par la transition sur la guerre : il s'agissait d'opposer la guerre et les fauteurs de guerre (en l'occurrence la gauche du Front populaire et, au présent, la gauche de gouvernement amalgamée avec le mouvement mémoriel), aux garants de la stabilité, de l'ordre et de la réconciliation nationale. Contre une historiographie universitaire accusée d'être partisane du Front populaire, ces plumes néofranquistes tenaient (et tiennent) le discours de l'objectivité historique, une objectivité appuyée sur des archives censées parler, là encore, d'elles-mêmes ${ }^{1}$. Difficile, en ayant en partage la défense de l'esprit de la transition et une conception objectiviste de l'histoire (quel que soit le degré de bonne foi de leurs adversaires), que les collègues ayant accepté le défi ne se retrouvent enferrés dans des discussions érudites infinies donnant l'impression au public d'un débat assez équilibré.

Pendant ce temps, prenant appui sur les signes extérieurs de légitimité académique que lui confère la structure de ses chapitres, Pío Moa a pu avancer très loin dans la formulation de positions ouvertement profranquistes. Amalgamant le travail des historiens sur la dictature avec l'antifranquisme militant, il les accuse de rejetter en bloc une période qu'il présente comme celle d'une décisive modernisation de l'Espagne, rejoignant un certain « sens commun $\gg$ qui est lui-même le résultat de la propagande et du discours de modernisation des dernières années du franquisme, mais aussi de l'absence de déconstruction de cette idéologie au moment de la transition. Les historiens, niant « la réalité » de l'industrialisation, l'alphabétisation, l'urbanisation, l'enseignement supérieur, la sécurité sociale attribuées à la dictature, sont qualifiés de « gens bien irrationnels », dont l'aveuglement et l'incapacité à expliquer la modernité de l'Espagne du présent vient de leurs origines militantes antifranquistes. Le dernier paragraphe du livre

(I) Une analyse des présupposés objectivistes en partage entre ces plumes d'extrême droite et l'historiographie classique de la guerre figure dans P. Sánchez León et J. Izquierdo Martín, La guerra que nos han contado..., op. cit. 
Los mitos de la guerra civil illustre jusqu'où il peut aller, à partir d'une fallacieuse neutralité :

«Il ne me paraît pas exagéré de dire, comme je l'ai déjà signalé dans De un tiempo y de un país, que la victoire de Franco lors de la guerre civile a sauvé l'Espagne d'une expérience révolutionnaire traumatisante, et que son régime lui a évité la guerre mondiale, a modernisé la société et a posé les bases d'une démocratie stable. En tenant compte de tous ses éléments négatifs et malgré l'image néfaste cultivée par ses ennemis ces dernières années, son bilan final me paraît très positif, et infondées la plupart des critiques qui circulent aujourd'hui comme des vérités incontestables ${ }^{1}$.»

En écrivant ses lignes, Pío Moa prend bien soin de ne pas franchir les règles morales fixées par la transition et étire jusqu'à l'extrême une position qui, par ailleurs, revendique le centre politique. Pleinement intégré et légitimé par des cercles médiatiques liés au Parti populaire, il fournit en retour une caution historique et une cohérence forte à un parti conservateur qui n'a pratiquement personne à sa droite.

Revendiqué à droite dans un mouvement de refondation d'identité politique, le patrimoine que représente le récit pacificateur de la guerre fratricide a pu l'être aussi à gauche, en réaction à ce premier mouvement mais aussi pour des raisons intrinsèques aux difficultés de représentation du Parti socialiste. Après la longue période pendant laquelle, dirigé par Felipe González, il avait résolument tourné le dos à l'histoire de la guerre conformément à l'esprit de la transition, une nouvelle ligne historiographique s'est développée. Après la perte de la majorité au profit du Parti populaire en 1996, la direction du Parti socialiste et en particulier celle de José Luis Zapatero a eu tendance à vouloir trouver dans la Seconde République

(I) P. Moa, Los mitos de la guerra civil..., op. cit. (p. 531). un esprit démocratique et progressiste, dans la continuité duquel elle jugeait important de se situer. Cette recherche de refondation identitaire était importante après que la politique appliquée pendant les trois mandats de Felipe González avait éloigné une bonne partie de l'électorat de gauche et elle pouvait être compatible avec l'idéal consistant à se situer « au centre » de l'échiquier politique, à condition de bien choisir ses antécédents dans le socialisme des années 1930. La figure de Juan Negrín incarnait «l'esprit de responsabilité » face à «l'aventurisme » et « l'inconséquence» d'un Francisco Largo Caballero, dirigeant ouvrier que sa tolérance pour l'antifascisme révolutionnaire rendait infréquentable $^{2}$. Negrín, lui, pouvait être paré de tous les attributs de l'homme d'État à stature internationale : son programme modéré et réconciliateur, et son activité diplomatique en direction de la Société des nations, du Comité de non-intervention et de la Grande-Bretagne en faisaient un «moderne » et même un « Européen ». Une fois lavé de l'accusation d'avoir été une marionnette du Parti communiste (accusation lancée par la gauche du Parti socialiste de l'époque, les anarchistes, les catalanistes et même d'autres républicains), il faisait un bel ancêtre. Autour de lui et de sa politique, une historiographie proche du Parti socialiste a même pu se développer alors que, pendant l'exil, elle avait à peine existé, ce parti ayant été une des grandes victimes de la guerre.

Cette figure est compatible avec une interprétation de la guerre «fratricide » formulée plus récemment, qui distingue trois Espagne, celles de la réforme, de la révolution et de la

(2) Francisco Largo Caballero fut le Premier ministre de la République en guerre entre septembre 1936 et mai 1937. Il représentait l'aile gauche du Parti socialiste, une culture et un discours politiques de la révolution ouvrière. Juan Negrín, son successeur jusqu'à la défaite, soutenu au départ par le « centre »du Parti socialiste ouvrier finit par s'appuyer surtout sur le Parti communiste. 
réaction, la première, majoritaire, ayant été victime des deux autres ${ }^{1}$. Dans une historiographie habitée par la recherche des responsables de ce qu'elle envisage d'abord comme une explosion de violence politique, il était essentiel d'écarter l'expérience partisane ou militante. Cela a contribué à forger l'idée, au cours des années 1990, d'une distinction nette entre, d'une part, des milieux de droite et de gauche imprégnés d'une «culture de la violence politique » au point de précipiter l'ensemble du pays dans la catastrophe et, d'autre part, une «majorité silencieuse » avant tout victime des premiers ${ }^{2}$. Une telle interprétation constitue une belle expression des valeurs cardinales de la monarchie constitutionnelle espagnole où prime la recherche de pactes et de consensus entre des partis « modernes » et où toute manière alternative de faire de la politique est moralement condamnée, disqualifiée comme $\ll$ violente $»^{3}$.

Dans le cadre du métarécit forgé pendant la transition, des avancées significatives sur le plan de la connaissance de la guerre sont réelles, comme en témoigne la récente et volumineuse synthèse dirigée par le plus éminent des historiens du conflit, Angel Viñas, par ailleurs auteur de la meilleure vue d'ensemble, en trois

(I) On trouve cette interprétation dans le livre d'Enrique Moradiellos, un des historiens les plus impliqués dans la polémique contre Pío Moa (Enrique Moradiellos, 1936 : los mitos de la guerra civil, Barcelone, Península, 2004). L'auteur appuie son interprétation sur une prise de distance modérée par rapport au métarécit transitionnel, sans pour autant se démarquer de l'idée dépolitisante d'une « guerre fratricide » dont il reconnaît « l'utilité fonctionnelle » mais aussi le caractère mythique et sacralisateur (p. 32).

(2) Jusqu'à la caricature chez Michael Seidman dans son livre Republic of Egos: A Social History of the Spanish Civil War, Madison, University of Wisconsin Press, 2002.

(3) Voir Pablo Sánchez León, «Violence, and the History and Memory of the Spanish Civil War: Beyond Inherited Narrative Frameworks on a Traumatic Past», in Manu Bragança et al. (dir.), Memories of the Second World War in Europe : Redifining the Self and the Nation, Londres, Berghahn Books, sous presse ; François Godicheau, « Hacer historia de la violencia », in Esther Pascua (dir.), ¿Qué hacemos con el pasado, la memoria y la bistoria?, Contratiempo, 2014. volets, sur la guerre d'Espagne ${ }^{4}$. Dans l'introduction de cet ouvrage collectif, Angel Viñas admet que sa structure est liée à une entreprise de rectification des faits face aux versions « déformées et profondément idéologiques » récemment éditées en Espagne. Bien que sa position consistant à considérer le binôme chronologique formé par la République et la guerre civile comme une tromperie, lui préférant la séquence de la guerre civile et de la dictature, contraste sensiblement avec le récit traditionnel, l'idée selon laquelle «défigurée durant plus de quarante ans de dictature franquiste, la dynamique interne du conflit est remontée à la surface de manière de plus en plus documentée $\gg$ continue à situer les principales réponses du côté de l'érudition plutôt que du côté des problèmes d'interprétation.

Sur ce plan, une nouveauté était pourtant apparue en 2006, sous la plume de l'historien Rafael Cruz, auteur d'En el nombre del Pueblo ${ }^{5}$. Armé des outils de la sociologie historique et de la théorie des mouvements sociaux, formé à l'école de Charles Tilly et de Sidney Tarrow, l'auteur offre une lecture tout à fait neuve du conflit, en des termes qui rendent beaucoup plus facile la comparaison avec d'autres pays. Il cesse par exemple d'utiliser le mot « répression $\gg$, directement hérité du conflit lui-même et souvent trop biaisé pour être utile à une

(4) Angel Viñas, En el combate por la historia : la república, la guerra civil y el franquismo, Barcelone, Pasado y Presente, 2012. Ce volume de 973 pages regroupe les travaux de trentequatre auteurs. Parmi les avancées signalées, on peut citer celles qui se rapportent à l'éclairage des conditions internationales qui firent la victoire franquiste (principalement la trilogie d'Angel Viñas lui-même, La soledad de la República : el abandono de las democracias y el viraje hacia la Unión soviética; El escudo de la República : el oro de España, la apuesta soviética y los hechos de mayo de 1937; El bonor de la República : entre el acoso fascista, la hostilidad británica y la política de Stalin, Barcelone, Crítica, 2006, 2007 et 2008) et à l'économie de l'Espagne en guerre (Enrique fuentes Quintana et Francisco Comín Comín, Economía y economistas españoles en la guerra civil, Madrid, Real Academia de ciencias morales y políticas, 2008, 2 vol.).

(5) Rafael Cruz, En el nombre del Pueblo, Madrid, Siglo XXI, 2006. 
histoire autre que victimaire, au profit de celui d'«épuration », qui permet la comparaison avec l'histoire des pays voisins et relie les actes de violence aux projets politiques qui les soustendent. Il avance l'hypothèse que la violence politique du printemps 1936 était avant tout liée à une reprise en main de l'ordre public par le gouvernement, qui préparait une coalition républicaine gauche-droite, rendue impossible par les événements de juillet. Ces propositions n'ont pourtant pratiquement pas fait l'objet de débat, sans doute parce qu'elles se démarquent du métarécit traditionnel, Rafael Cruz refusant par exemple d'utiliser l'expression « guerre civile ${ }^{1}$. Si ce métarécit est un patrimoine politique essentiel du régime issu de la transition, cette absence de débat ou de renouvellement interprétatif n'est pas si étonnante. Même si les conditions de production de l'historiographie ont des conséquences profondes sur les pratiques des historiens, un changement de paradigme historiographique peut difficilement venir d'eux seuls, quelle que soit la qualité de leur travail. La crise générale que vit actuellement l'Espagne, qui se traduit notamment par une mise en cause très sérieuse des institutions nées en 1978, elle-même liée à des mouvements sociaux d'un nouveau genre, à un phénomène générationnel certain et à la revendication culturelle du «mouvement de la mémoire » qui a été une sorte de premier pas, est sans doute plus susceptible de créer les conditions d'un changement de paradigme dans le rapport de l'Espagne aux récits de son passé récent.

François Godicheau, Université Bordeaux-III, Sciences Philosophie Humanités (SPH), 33607, Pessac cedex, France.

François Godicheau est professeur à l'Université BordeauxIII et membre junior de l'Institut universitaire de France (IUF). II a publié plusieurs ouvrages sur la guerre d'Espagne, dont $L a$ Guerre d'Espagne, république et révolution en Catalogne (Odile Jacob, 2004), La Guerre d'Espagne, de la démocratie à la dictature (Gallimard, 2006) et No callaron : las voces de los presos antifascistas en las cárceles republicanas (Presses universitaires du Mirail, 2012) ainsi que de nombreux articles. Travaillant à présent essentiellement sur le $19^{\mathrm{e}}$ siècle, il poursuit néanmoins une réflexion sur l'historiographie du $20^{\mathrm{e}}$ siècle espagnol et a récemment dirigé le livre Democracia Inocua : lo que el posfranquismo ha hecho de nosotros (Contratiempo, 2014). (Francois.Godicheau@u-bordeaux3.fr)
(I) Un autre livre très important sur la construction de la figure de l'ennemi, qui ne cadre pas avec le récit traditionnel, n'a pas été intégré à l'histoire générale du conflit : Nuñez Seixas et Xosé Manuel, ;Fuera el invasor! Nacionalismos y movilización bélica durante la guerra civil española (1936-1939), Madrid, Marcial Pons, 2006. 